\title{
Can a supervised algorithmic assessment of men for prostate cancer improve the quality of care? A retrospective evaluation of a prostate assessment pathway in Saskatchewan
}

\author{
Bonnie Liu'; Kunal Jana, MD2; Gary Groot, $M D^{3}$ \\ 'College of Medicine; '2Department of Urology; '3epartment of Community Health and Epidemiology; University of Saskatchewan, Saskatoon, SK, Canada
}

Cite as: Can Urol Assoc J 2017;11 (9):E338-43. http://dx.doi.org/10.5489/cuai.4237

Published online September 12, 2017

\section{Abstract}

Introduction: The Saskatoon Prostate Assessment Pathway (SPAP) was developed in 2013 in part to decrease the wait times between physician referral and biopsy for patients with suspected prostate cancer. Using an algorithm carefully designed to optimize appropriate prostate biopsy rates, physicians can directly refer patients for biopsy through the SPAP without seeing a urologist. All other patients are referred to the Saskatoon Urology Associates (SUA). The present study evaluates the performance of the algorithm.

Methods: 971 patients seen at the SUA and 302 patients seen through the SPAP were identified. Information on age, biopsy status and outcome, risk stratification, and time between referral and biopsy was collected. Biopsy wait time data was analyzed using gamma distribution. Association between referral method and biopsy rate, and between referral method and risk stratification, was analyzed using Z-test.

Results: The expected wait time from referral to biopsy for patients seen through SUA was 2.63 times longer than those seen through SPAP (34 vs. 91 days). The biopsy rate of patients seen in the SPAP was significantly higher than those by SUA (88\% vs. $69 \%, 95 \%$ confidence interval [Cl] 0.14-0.26; $\mathrm{p}<0.00001)$. There was no significant difference in positive biopsy rates for patients seen through the SPAP vs. SUA (81\% vs. $74 \%, 95 \% \mathrm{Cl}-0.011,0.14 ; \mathrm{p}=0.095)$, for detection of low-risk cancer, (12\% vs. $10 \%, 95 \% \mathrm{Cl}-0.034,0.080$; $\mathrm{p}=0.44)$, or for clinically relevant cancer, i.e., intermediate- and high-risk cancer, for SPAP vs. SUA $(56.54 \%$ vs. $56.68 \%, 95 \% \mathrm{Cl}$ -0.091,0.089; $p=0.49$ ).

Conclusions: The algorithm used in the SPAP is effective in decreasing wait time to prostate biopsy and has the same cancer/pre-cancer detection rate, but at the cost of a higher biopsy rate. Both referral mechanisms result in few low-risk cancer detection biopsies, finding primarily cases of high- or intermediate-risk cancer.

\section{Introduction}

While the use of prostate specific antigen (PSA) screening to detect prostate cancer is controversial, ${ }^{1-3}$ the reality is that prostate cancer screening is still widely practised. Moreover, the Canadian Strategy for Cancer Control recommends that the time between presentation to provider with possible cancer and definitive diagnosis should be less than four weeks, ${ }^{1}$ yet the median diagnostic interval for prostate cancer in Canada is 53 days. $^{2}$ Although the evidence on the impact of wait times on prostate cancer mortality is inconclusive, diagnosis and treatment delays have been shown to impact patient and family psychosocial distress. ${ }^{3-6}$ The Saskatoon Prostate Assessment Pathway (SPAP) was established with several goals in mind, one of which was to reduce the time from physician referral to biopsy, thus shortening the diagnostic wait time. This study does not assess the appropriateness of using PSA as a screening tool or whether prostate cancer screening should be performed. Instead, it was undertaken to evaluate the effect of the SPAP on diagnostic wait time, with the understanding that once patients have been screened for prostate cancer by their healthcare provider, appropriate followup steps need to be taken to ensure appropriate care is delivered. The other goals of the SPAP (reducing practice variation between urologists and improving patient decision-making) were not evaluated in this study.

The pathway is administered through the Leslie and Irene Dubé Urology Centre of Health at St. Paul's Hospital, which opened in 2013 in conjunction with the introduction of the SPAP. The pathway allows primary care providers to refer men directly for a prostate biopsy provided they meet the requirements of an algorithm designed to capture the factors that influence a urologist's decision to recommend a biopsy or not. The algorithm used by the Centre was developed through a series of evidence-informed consensus-building meetings of a multidisciplinary group. The group included patients, urologists, family physicians, radiation oncologists, and a nurse navigator. It includes parameters around age, age-specific 
PSA cutoffs, digital rectal exam (DRE) findings, previous prostate biopsies, life expectancy, and comorbidities. The SPAP is under the oversight of a urologist, but depending on biopsy outcomes and the treatment decisions of the patient, he may never come into contact with a urologist.

The primary objective of this study was to evaluate the performance of the algorithm used in the SPAP with regards to the aspect of diagnostic wait times. We assessed the SPAP for diagnostic reliability (does the algorithm reliably select patients for biopsy compared with a urologist?), efficacy (does the algorithm biopsy the same number of patients as a urologist to achieve the same results?), and efficiency (does use of the algorithm decrease wait times to diagnosis compared to a urologist referral?).

\section{Methods}

After obtaining ethics exemption (reference REB: BIO-1698), we collected data for patients seen between May 2013 and October 2015. Data from the urology referral group was collected by generating a list of patients through diagnostic billing codes for abnormal PSA, with or without a prostate nodule, and performing a retrospective chart review. Of the 1172 patients on the generated list, only patients who fulfilled the inclusion criteria were included in the Saskatoon Urology Associates (SUA) data (Table 1). This produced a cohort of 971 patients that were included in the study; 31 patients were seen by a urologist, discharged from the urologist's care, then re-referred, resulting in total of 1002 data points. Patients were then categorized as SPAP-eligible or not (Table 2), and only patients who were pathway-eligible were used to compare study outcomes.

Patients are referred to the SPAP through a referral form sent to the Urology Centre, and a pathway-eligible patient is seen by a specialized nurse navigator who explains the biopsy procedure and helps the patient determine if he wants to proceed with a biopsy. Patients agreeing to a biopsy will then make an appointment to receive one without having to consult a urologist first. Any patient who is referred to the

\begin{tabular}{lcc}
\hline Table 1. Study exclusion criteria & & \\
\hline & SPAP & SUA \\
\hline $\begin{array}{l}\text { Total number of patients reviewed } \\
\text { Exclusion criteria }\end{array}$ & 372 & 1172 \\
$\quad \begin{array}{l}\text { Existing/previous cancer diagnosis } \\
\quad \text { Seen by urologist/nurse navigator outside }\end{array}$ & 7 & 110 \\
$\quad \begin{array}{l}\text { timeframe (May 2013-October 2015) } \\
\text { Incorrect billing code entered/not actually seen }\end{array}$ & 1 & 31 \\
$\quad \begin{array}{l}\text { for abnormal PSA or DRE } \\
\text { Ineligible SPAP patients never seen by urologist }\end{array}$ & 40 & - \\
$\quad$\begin{tabular}{l} 
Total excluded \\
\hline DRE: digital rectal exam; PSA: prostate-specific antigen; SPAP: Saskatoon Prostate
\end{tabular} & \\
\hline Assessment Pathway; SUA: Saskatoon Urology Associates. & & \\
\hline
\end{tabular}

SPAP but found to be pathway-ineligible, or who declines a biopsy, is automatically referred to the SUA for followup with a urologist. Fig. 1 outlines the flowchart for patient categorization. Data for the SPAP referral group was collected from prospective data maintained by staff at the Urology Centre. There were 372 patients referred to the SPAP within our timeframe, of which 302 fulfilled the inclusion criteria of the study (Table 1).

Data collected included patient age, pathway eligibility, whether they received a biopsy, time between referral and biopsy, and the outcome and risk stratification of the biopsy. Biopsies were performed by a radiologist in Saskatoon. Risk stratification was based on the D'Amico risk stratification system. ${ }^{7}$ Positive biopsies included those that showed cancer or atypical small acinar proliferation (ASAP), since ASAP is associated with a significant risk of developing prostate cancer. ${ }^{8}$

Table 2. Saskatoon Prostate Assessment Pathway (SPAP)ineligible patients

\begin{tabular}{|c|c|}
\hline \multicolumn{2}{|c|}{ Question } \\
\hline \multicolumn{2}{|c|}{$\begin{array}{l}\text { Patient under } 40 \text { years } \\
\text { or over } 75 \text { years with } \\
\text { elevated PSA }\end{array}$} \\
\hline \multicolumn{2}{|c|}{$\begin{array}{l}\text { Patient has PSA value } \\
\text { greater than } 50\end{array}$} \\
\hline \multicolumn{2}{|c|}{$\begin{array}{l}\text { PSA values less than } \\
\text { normal age-specific PSA } \\
\text { levels }\end{array}$} \\
\hline Age & PSA (upper limit) \\
\hline $40-49$ & $2.5 \mathrm{ng} / \mathrm{ml}$ \\
\hline $50-59$ & $3.5 \mathrm{ng} / \mathrm{ml}$ \\
\hline $60-69$ & $4.5 \mathrm{ng} / \mathrm{ml}$ \\
\hline $70+$ & $6.5 \mathrm{ng} / \mathrm{ml}$ \\
\hline
\end{tabular}

Patient has abnormal DRE, but PSA value is normal

\section{Rationale}

Patients under 40 years and over

75 years require further assessment to determine if a prostate biopsy is necessary. If a patient is over 75 years, a prostate biopsy may not be appropriate

If a patient has a PSA value of greater than 50 the diagnosis of prostate cancer may be based on clinical findings and a prostate biopsy may be avoided

PSA levels are subject to considerable variation, and increase with age. Two abnormal PSA levels at least six weeks apart are required prior to referral. PSA screening

for men over 70 years is not recommended

If a patient has an abnormal DRE and the PSA value is normal, they require a urologist to assess the prostate to determine if the abnormality warrants a biopsy

With a previous negative prostate Patient has had a biopsy subsequent biopsies may not previous biopsy be necessary because the change of a positive finding is less likely with each subsequent biopsy

Patient is immunocompromised

If a patient is immunocompromised additional preparation may be required prior to a prostate biopsy

If a patient has a life expectancy of less than 10 years further

Patient life expectancy less than 10 years assessment/treatment may not be appropriate

DRE: digital rectal exam (taken from the Saskatoon Prostate Assessment Pathway (SPAP) referral form); PSA: prostate-specific antigen. 


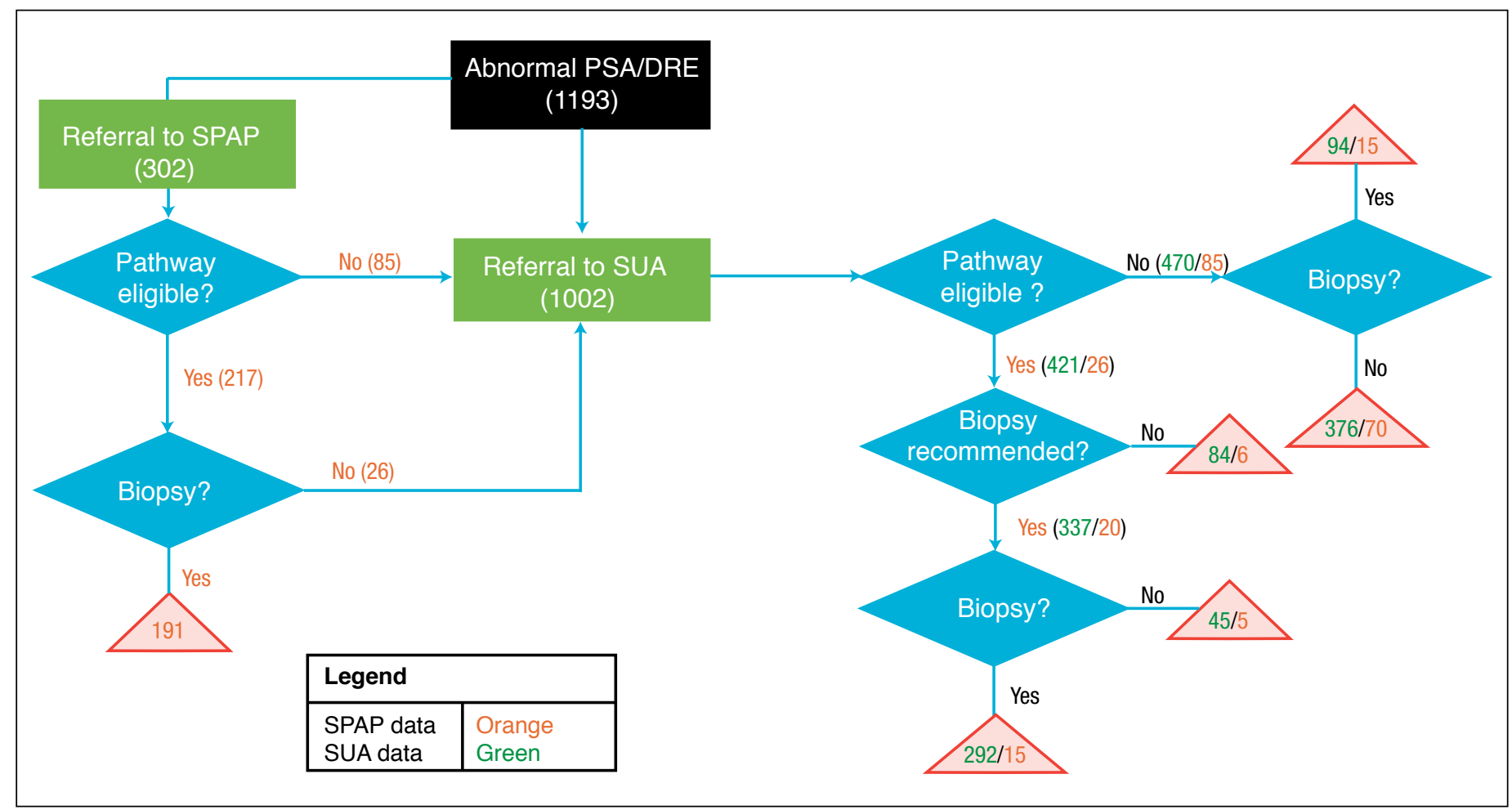

Fig. 1. Flowchart outlining patient referral mechanism for followup. Only patients who were included in the study are shown. Patients referred to Saskatoon Prostate Assessment Pathway (SPAP) who were pathway-ineligible or eligible but did not get a biopsy for whatever reason were automatically referred to Saskatoon Urology Associates (SUA) and seen by a urologist for further assessment. DRE: digital rectal exam; PSA: prostate-specific antigen.

The primary outcome of time between referral and biopsy was assessed using gamma distribution, as the data was skewed to the right and not normally distributed (Fig. 2). The difference in age was analyzed using Z-test. The association between referral method and biopsy rate, positive

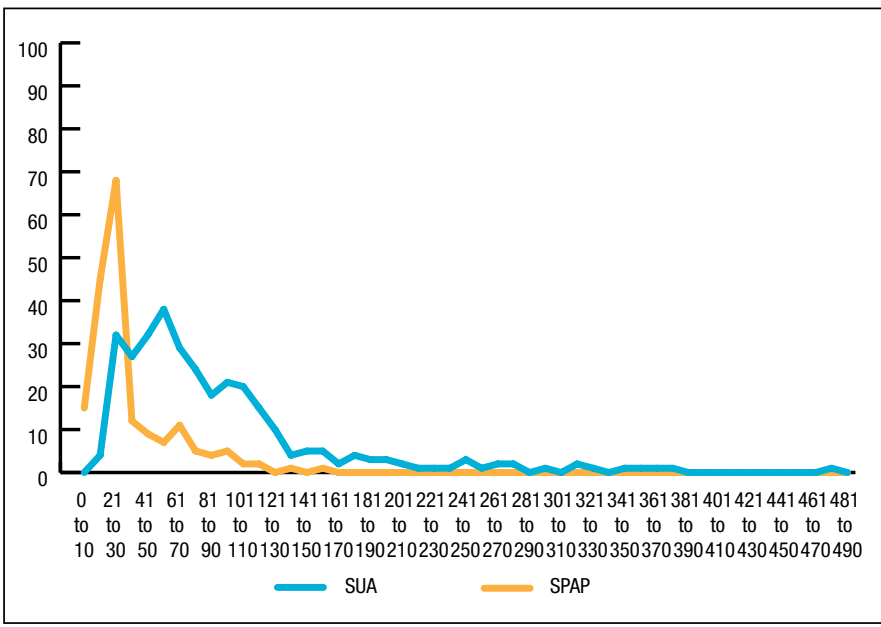

Fig. 2. Wait time between physician referral and biopsy. Patients seen through Saskatoon Urology Associates (SUA) had an expected time between referral and biopsy of 91 days, whereas patients seen through Saskatoon Prostate Assessment Pathway (SPAP) had an expected wait time of 34 days. The expected time was calculated based on gamma distribution, as the data is nonnormal and has a right skew. cancer findings, and resulting risk stratification was analyzed using Z-test. A critical value of 1.96 for $5 \%$ two-tailed was considered statistically significant.

\section{Results}

\section{Demographics}

There was no statistically significant difference in average age between patients seen in the pathway compared to those seen by urologists (64 vs. 63 years; 95\% confidence interval $[\mathrm{Cl}]$ $0.21-1.91 ; \mathrm{p}=0.058)$ (Table 3).

\section{Time difference}

For pathway-eligible patients seen through the SPAP, the expected time between referral and biopsy, based on gamma distribution calculations, was 34.47 days $(\alpha=2.28, \beta=15.12)$. $\mathrm{Q}_{1}$ was 17.73 days, the median was 29.64 days, $\mathrm{Q}_{3}$ was 46.06 days, and the interquartile range (IQR) was 28.33 (Table 4). The expected time for pathway-eligible patients seen by SUA was 90.72 days $(\alpha=2.28, \beta=15.12)$, with a $Q_{1}$ of 43.81 days, a median of 76.24 days, $\mathrm{Q}_{3}$ of 122.02 days, and an IQR of 78.21 (Table 4). Expected time comparison between SPAP and SUA showed that patients were seen 2.63 times faster 
Table 3. Age demographics

\begin{tabular}{|c|c|c|c|c|}
\hline & SPAP & SUA & $95 \% \mathrm{Cl}$ & p \\
\hline Age (years) & 64 & 63 & $-0.21-1.91^{*}$ & 0.058 \\
\hline
\end{tabular}

through SPAP. SPAP expected time was $38 \%$ that of SUA expected time.

\section{Biopsy rate}

Of the 302 patients referred to the SPAP, 217 were determined to be pathway-eligible and 191 were biopsied. Of the 1002 patients referred to SUA, 447 were determined to be pathway-eligible and 307 of them were biopsied. Reasons for not getting a biopsy included factors such as patient preference, a significant drop in PSA, and an elevated but stable PSA. The biopsy rate of patients seen in the SPAP was significantly higher than those of SUA ( $88 \%$ vs. $69 \% ; 95 \% \mathrm{Cl}$ $0.14,0.26 ; p<0.00001$ ) (Table 5, Fig. 3). Any patient seen in the SPAP who refused biopsy was directed to SUA and some patients continued to refuse biopsy when seen by the urologist. To account for this, all patients with the final decision of declining the recommended biopsy were removed from the sample (36 patients), as their decision would likely be the same regardless of the referral mechanism and thus may bias the data. The biopsy rate of patients seen in the SPAP was still significantly higher than those seen by SUA $(88 \%$ vs. $75 \% ; 95 \% \mathrm{Cl} 0.082,0.20 ; \mathrm{p}<0.0001)$. There were also 13 patients seen by SUA who were recommended a biopsy, but were lost to followup and never received the biopsy. When these patients were removed from the sample, the biopsy rate of patients seen in SPAP remained significantly higher than those seen by SUA (88\% vs. $77 \% ; 95 \%$ Cl 0.059, 0.18; $\mathrm{p}=0.0001$ ) (Table 5, Fig. 3).

\section{Biopsy outcome and risk stratification}

There was no significant difference in positive biopsy outcomes between the SPAP and SUA (81\% vs. 74\%; 95\% $\mathrm{Cl}-0.011,0.14 ; \mathrm{p}=0.095)$ (Table 5, Fig. 4A). No significant difference was found in detection of ASAP (12\% vs. $8 \%$; $95 \% \mathrm{Cl}-0.020,0.091 ; \mathrm{p}=0.10)$ or low-risk cancer, $(12 \%$ vs. $10 \% ; 95 \% \mathrm{Cl}-0.034,0.080 ; \mathrm{p}=0.44$ ) (Table 5, Fig. 4B). Intermediate-risk results were significantly higher in the SPAP patients ( $38 \%$ vs. $27 \%$ [SPAP vs. SUA]; $95 \% \mathrm{Cl} 0.025$, 0.19; $p=0.0055)$, while SUA patient has significantly higher detection rates of high risk cancer $(19 \%$ vs. $30 \%$ [SPAP vs. $\mathrm{SUA}$; $95 \% \mathrm{Cl}-0.19$, -0.036; $\mathrm{p}=0.0020)$; however, when intermediate- and high-risk cancers were grouped, as these are the more clinically relevant cancers, there was no significant difference in detection rates for SPAP vs. SUA ( $57 \%$ vs. $57 \% ; 95 \% \mathrm{Cl}-0.091,0.089 ; \mathrm{p}=0.49$ ) (Table 5, Fig. 4B).

$\begin{aligned} & \text { Table 4. Time from referral to biopsy (days), calculated } \\
& \text { based on gamma distribution }\end{aligned}$
\begin{tabular}{llc}
\hline Variable & SPAP & SUA \\
\hline Expected time* & $34.47^{\infty}$ & $90.72^{* *}$ \\
Q1 & 17.73 & 43.81 \\
M & 29.64 & 76.24 \\
Q3 & 46.06 & 122.02 \\
IOR & 28.33 & 78.21 \\
\hline
\end{tabular}

Q1 is the first quartile, encompassing $25 \%$ of the data set; $M$ is the median, encompassing $50 \%$ of the data set; $\mathrm{Q} 3$ is the third quartile, encompassing $75 \%$ of the data set; and interquartile range [IOR] = Q3-Q1; ${ }^{*}$ expected time is calculated by multiplying $\alpha$ and $\beta$; ${ }^{* *}$ SUA: $\alpha=2.01, \beta=44.91$; ${ }^{\infty}$ SPAP: $\alpha=2.28, \beta=15.12$. SPAP: Saskatoon Prostate Assessment Pathway; SUA: Saskatoon Urology Associates.

\section{Discussion}

Several factors, such as age, PSA, DRE findings, and outcomes of previous biopsies have been identified in the literature to predict the likelihood of finding prostate cancer with a prostate biopsy. ${ }^{9}$ These factors together have been shown to be superior at estimating the risk of prostate cancer when compared to using PSA levels alone. ${ }^{3}$ Finne et al used regression models to show that multivariate algorithms can be used to reduce unnecessary prostate biopsies compared to using free PSA alone..$^{10}$ To our knowledge, ours is the first study to evaluate an algorithm used in practice by comparing biopsy rates between urologists and the algorithm. Our results show that the algorithm helps shorten the time to biopsy by over $60 \%$ and produces the same positive biopsy rate, but at the cost of more biopsies. This difference in biopsy rate remained even when removing all patients who refused biopsy, as their biopsy decision is likely unchanged regardless of which referral mechanism they go through. A significant difference was again apparent when also removing patients lost to followup, which was only the case in patients seen through SUA. Importantly, the algorithm did not result in more low-risk or ASAP prostate

\begin{tabular}{|c|c|c|c|c|}
\hline & SPAP & SUA & $95 \% \mathrm{Cl}$ & $\mathbf{p}$ \\
\hline Biopsy rate (\%) & 88.18 & 68.68 & $0.14,0.26$ & $<0.00001$ \\
\hline $\begin{array}{l}\text { Biopsy rate, removing } \\
\text { patients who declined } \\
\text { and lost to followup (\%) }\end{array}$ & 88.18 & 77.14 & $0.059,0.18$ & 0.0001 \\
\hline $\begin{array}{l}\text { Positive biopsy finding } \\
(\%)\end{array}$ & 80.41 & 74.26 & $-0.011,0.14^{*}$ & 0.095 \\
\hline ASAP (\%) & 12.04 & 8.47 & $-0.20,0.091^{*}$ & 0.10 \\
\hline Low-risk (\%) & 12.04 & 9.77 & $-0.034,0.080^{*}$ & 0.44 \\
\hline Intermediate-risk (\%) & 37.70 & 26.72 & $0.025,0.19$ & 0.0055 \\
\hline High-risk (\%) & 18.85 & 29.97 & $-0.19,-0.036$ & 0.0020 \\
\hline $\begin{array}{l}\text { Clinically significant } \\
\text { (intermediate and } \\
\text { high risk) (\%) }\end{array}$ & 56.54 & 56.68 & $-0.091,0.0088^{*}$ & 0.49 \\
\hline \multicolumn{5}{|c|}{$\begin{array}{l}\text { 'The difference in positive biopsy finding was non-significant ( } p>0.05) \text {. Risk stratification is } \\
\text { based on the D'Amico risk stratification system. ASAP: atypical small acinar proliferation; } \\
\text { Cl: confidence interval; SPAP: Saskatoon Prostate Assessment Pathway; SUA: Saskatoon } \\
\text { Urology Associates. }\end{array}$} \\
\hline
\end{tabular}




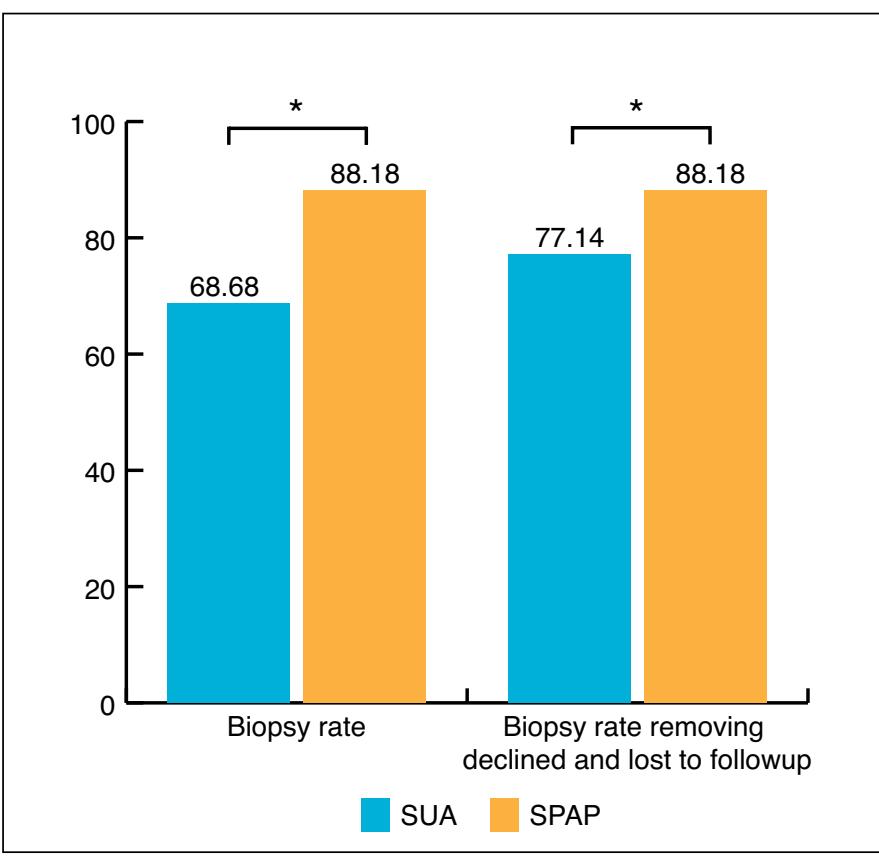

Fig. 3. Biopsy rates for patients seen either by the Saskatoon Urology Associates (SUA) or the Saskatoon Prostate Assessment Pathway (SPAP). The right side of the graph shows biopsy rates after patients who declined biopsy were removed, as it is assumed that regardless of which referral mechanism the patient went through, he would refuse biopsy. Patients who were lost to followup were also removed from the sample for the right side of the graph, as it is unclear what their outcome would have been had they been followed up on. ${ }^{*} \mathrm{p}<0.05$.

biopsies, and was as effective as urologists in the detection of more clinically significant prostate cancers.

The time benefit of the SPAP over SUA referrals should not be underestimated. One-fifth to half of cancer patients identify cancer diagnosis as a major source of distress and suspected cancer patients may be more negatively affected by anxiety compared to cancer patients with confirmed diagnoses. ${ }^{5}$ Men with prostate cancer in Canada have longer wait times for diagnosis and treatment than other cancers ${ }^{2,6}$ and prolonged wait times for cancer diagnosis and treatment have been associated with increased anxiety, distress, and a feeling of powerlessness. ${ }^{6}$ There is evidence that it is the uncertainty about their prostate cancer that causes men the most distress ${ }^{11,12}$ and the faster they can move on to diagnosis and treatment, the better they are able to cope with their illness. ${ }^{13}$ Biopsy results, even when they are not the preferred outcome, have been shown to have a general anxiety relief value for men with elevated PSA. ${ }^{11}$ This is an important factor to consider because distress can both increase healthcare costs associated with cancer, as well as reduce adherence to treatment. ${ }^{13}$ Patients referred by their family physician to SPAP had an expected wait time that was 56 days shorter than for patients seen by SUA. Our assumption is that the pathway can help patients reduce their anxiety levels and

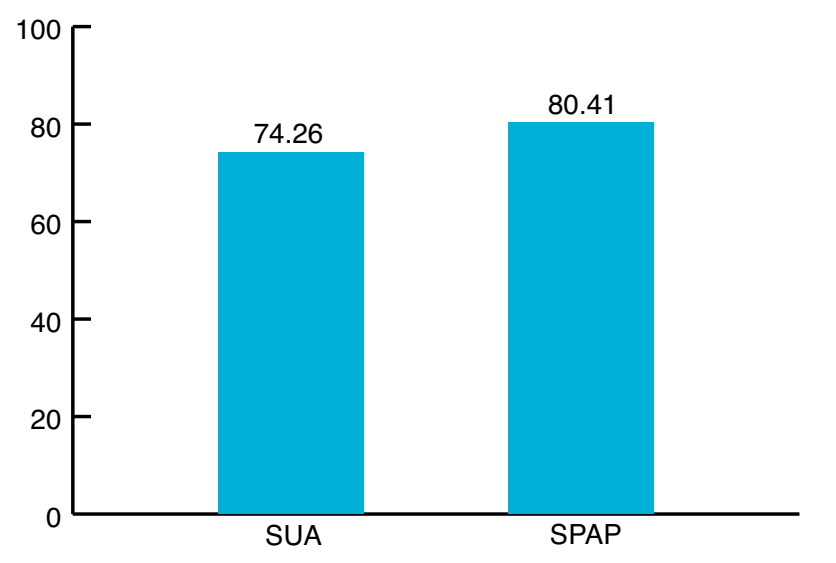

Fig. $\mathbf{4 A}$. Biopsy results showing positive findings. Positive findings include pathology results of cancer (low-, intermediate- or high-risk stratification), or atypical small acinar proliferation (ASAP), as these cells are more likely to eventually progress to prostate cancer. SPAP: Saskatoon Prostate Assessment Pathway; SUA: Saskatoon Urology Associates.

result in better health outcomes, although further qualitative study is needed to confirm this.

Although the biopsy rates were higher in the patients seen by SPAP, there was no significant difference in positive biopsy rates compared to patients seen by SUA. Notably, there was no significant difference in the detection rates of more clinically relevant cancer (i.e., intermediate- and highrisk) and low-risk prostate cancer. This, combined with the high cancer detection rates of $81 \%$ and $74 \%$ for SPAP and SUA, respectively, show that there is validity in using the criteria set by the algorithm; however, there might be room for further refinement of the algorithm to capture aspects of decision-making by urologists that might lead to a further decrease in the number of unnecessary biopsies. Even with adjustments, the algorithm will likely not be able to catch all patients with prostate cancer, since many patients with prostate cancer have a normal DRE and low PSA levels, ${ }^{14}$ but it will most certainly allow physicians to diagnose the majority of new prostate cancers without having to biopsy all men. Detection rates may improve with the advent of novel biomarkers, such as kallikrein markers and some urinary biomarkers, which have shown some promise in better prostate cancer detection rates when compared to PSA testing. ${ }^{15}$ Should these markers prove effective, they may also be added as a component of the algorithm. Additional adjustment may come from analysis of urologist practice variation. Preliminary data on variation in practice between urologists has been collected and additional study is required determine the role that practice variation has on prostate cancer biopsies and outcomes. Integrating aspects of the best performing urologists into the algorithm and reducing unwarranted variation among urologists would potentially strengthen the value of this tool for patients overall. 


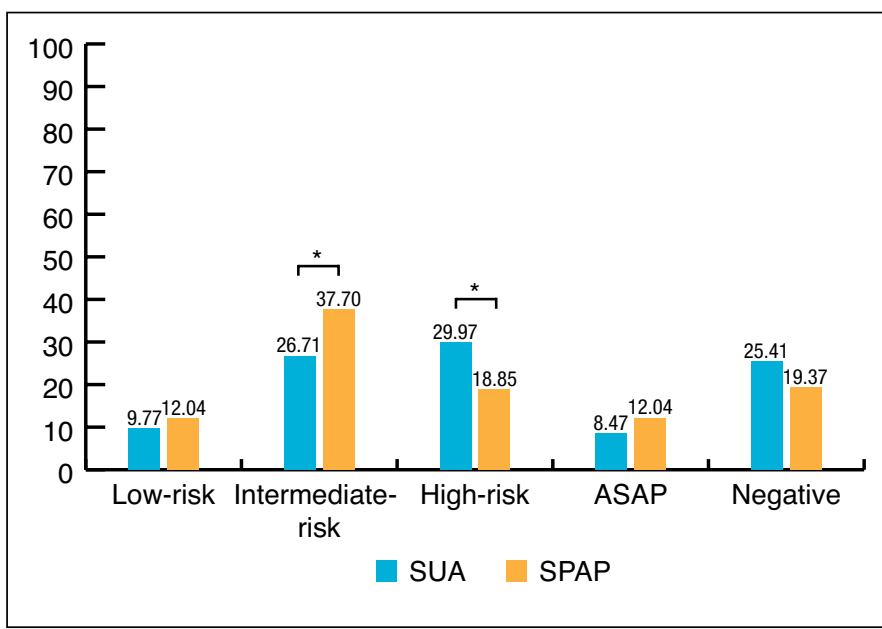

Fig. 4B. Risk stratification for patients who underwent a biopsy. ${ }^{*} \mathrm{p}<0.05$. SPAP: Saskatoon Prostate Assessment Pathway; SUA: Saskatoon Urology Associates.

\section{Limitations}

One limitation of the study is that the collection of patient information for this study was based on diagnostic codes entered by SUA office staff. Patients who were incorrectly entered would not have been captured in this study. Data regarding serum PSA at referral, DRE findings by primary care provider, DRE finding by urologist, family history of prostate cancer, and race were not collected. While these factors may affect prostate cancer development and detection, they fell outside the scope of this current study. It should also be noted that we do not have long-term data on patients who did not receive a biopsy through either the SUA or SPAP, thus do not know how many of those patients went on to develop prostate cancer.

\section{Conclusion}

The evidence-informed algorithm used by the SPAP is effective in decreasing wait time to prostate biopsy and has the same cancer/pre-cancer detection rate, but at the cost of a significantly higher biopsy rate; however, the algorithm has the same detection rate for clinically significant cancer and does not increase the number of inappropriate biopsies when compared to urologists. Careful consideration is necessary when balancing the benefits of reduced wait time to diagnosis with the increased biopsy rate to achieve the same results. Refinement of the algorithm to improve its performance might be a future consideration.

Competing interests: Dr. Groot has been a speaker for Sanofi and has participated in a clinical trial supported by $\mathrm{KCl}$ Canada. The remaining authors report no competing personal or financial interests.

This paper has been peer-reviewed.

\section{References}

1. Canadian Strategy for Cancer Control Governing Council. Canadian Strategy for Cancer Control: A Cancer Plan for Canada. Health Canada 2006.

2. Stevens C, Bondy SJ, Loblaw DA. Wait times in prostate cancer diagnosis and radiation treatment. Can Urol Assoc J 2010;4:243-8. https://doi.org/10.5489/cuaj.09122

3. Schröder F, Kattan MW. The comparability of models for predicting the risk of a positive prostate biopsy with prostate-specific antigen alone: A systematic review. Eur Urol 2008;54:274-90. https://doi.org/10.1016/i.eururo.2008.05.022

4. Finne P, Finne R, Bangma C, et al. Algorithms based on prostate-specific antigen (PSA), free PSA, digital rectal examination, and prostate volume reduce false-positive PSA results in prostate cancer screening. Int J Cancer 2004;111:310-5. hitps://doi.org/10.1002/iic.20250

5. Brocken P, Prins JB, Dekhuïzen PNR, et al. The faster the better? A systematic review on distress in the diagnostic phase of suspected cancer, and the influence of rapid diagnostic pathways. Psychooncology 2012;21:1-10. https://doi.org/10.1002/pon.1929

6. Tran K, Sandoval C, Rahal R, et al. Wait times for prostate cancer treatment and patient perceptions of care in Canada: A mixed-methods report. Curr Oncol 2015;22:361-4. https://doi.org/10.3747/c0.22.2795

7. D'Amico AV, Whittington R, Malkowicz SB, et al. Biochemical outcome after radical prostatectomy, external beam radiation therapy, or interstitial radiation therapy for clinically localized prostate cancer. JAMA 1998;280:969-74. https://doi.org/10.1001/jama.280.11.969

8. Montironi R, Scattoni V, Mazzucchelli R, et al. Atypical foci suspicious but not diagnostic of malignancy in prostate needle biopsies (also referred to as "atypical small acinar proliferation suspicious for but not diagnostic of malignancy"). Eur Urol 2006;50:666-74. https://doi.org/10.1016/i.eururo.2006.07.048

9. Roobol MJ, Carlsson SV. Risk stratification in prostate cancer screening. Nat Rev Urol 2012;10:38-48. https://doi.org/10.1038/nrurol.2012.225

10. Finne P, Finne R, Bangma C, et al. Algorithms based on prostate-specific antigen (PSA), free PSA, digital rectal examination and prostate volume reduce false-positive PSA results in prostate cancer screening. Int J Cancer 2004;111:310-5. hitps://doi.org/10.1002/iic.20250

11. Dale W, Bilir $P$, Han $M$, et al. The role of anxiety in prostate carcinoma: a structured review of the literature. Cancer 2005;104:467-78. https://doi.org/10.1002/cncr.21198

12. Fitch MI, Gray RE, McGowan T, et al. Travelling for radiation cancer treatment: Patient perspectives. Psychooncology 2003;12:664-74. https://doi.org/10.1002/pon.682

13. Baker P, Beesley H, Dinwoodie R, et al. "You're putting thoughts into my head': A qualitative study of the readiness of patients with breast, lung, or prostate cancer to address emotional needs through the first 18 months after diagnosis. Psychooncology 2013;22:1402-10. https://doi.org/10.1002/pon.3156

14. Thompson IM, Pauler DK, Goodman PJ, et al. Prevalence of prostate cancer among men with a prostatespecific antigen level $\leq 4.0 \mathrm{ng} / \mathrm{ml}$. N Engl J Med 2004;350:2239-46. https://doi.org/10.1056/ NEJMoa031918

15. Scher HI, Scardino PT ZM. Chapter 68: Cancer of the Prostate. In: DeVita, Hellman, and Rosenberg's Cancer: Principles and Practice of Oncology 10th ed. Philidelphia, Pa: Lippincott Williams \& Wilkins; 2015

Correspondence: Dr. Kunal Jana, Department of Urology, University of Saskatchewan, Saskatoon, SK, Canada; janak@saskatoonhealthregion.ca 